\title{
Direct Solid-State Nucleation From Preexisting Coherent Precipitates in Aluminium
}

\author{
Laure Bourgeois ${ }^{1}$, Yiqiang Chen ${ }^{2}$, Zezhong Zhang ${ }^{2}$, Yong Zhang ${ }^{2}$ and Nikhil Medhekar ${ }^{2}$ \\ 1. Monash Centre for Electron Microscopy and Department of Materials Science and Engineering, \\ Monash University, Victoria, Australia. \\ 2. Department of Materials Science and Engineering, Monash University, Victoria, Australia.
}

Precipitation hardening is responsible for the strongest aluminium alloys currently in service. It usually involves the precipitation of metastable phases from a supersaturated solid solution. A textbook case is the precipitation of Guinier-Preston (GP) zones in Al-Cu alloys [1] and the subsequent formation of $\theta^{\prime \prime}$ and $\theta^{\prime}$ metastable phases [2]. More than 75 years after the original work by Guinier and Preston, the atomic-scale mechanisms behind the solid-solid phase transformations associated with the nucleation and growth of those phases remain unknown. One aspect of phase transformations that was proposed many decades ago [3] but never demonstrated, is the direct nucleation of a phase from its coherent precipitate precursor. In this contribution we show that this particular phase transformation is indeed possible and can be brought about in different ways.

We used an aberration-corrected FEI Titan ${ }^{3} 80-300$ at $300 \mathrm{kV}$ in high-angle annular dark field scanning transmission electron microscopy (HAADF-STEM) mode to image solid-state precipitates in Al1.7at.\%Cu, Al-1.7at.\%Ag and Al-1.7at.\%Cu-0.02at.\%Au alloys. The images shown were not processed apart from minor brightness-contrast adjustments.

Figure 1 shows HAADF-STEM images of $\theta^{\prime}$ phase nuclei having nucleated within a coherent precipitate of $\theta^{\prime \prime}$ phase. The latter phase consists of parallel $\mathrm{Cu}$ layers separated by three $\mathrm{Al}\{002\}$ layers. This nucleation is brought about by the incorporation of $\mathrm{Au}$ atoms within the $\theta^{\prime}$ structure and the subsequent lowering of the formation energy, as calculated by density functional theory [4]. The $\theta^{\prime}$ phase is an efficient strengthening phase of light $\mathrm{Al}$ alloys, and its enhanced nucleation rate results in a significantly stronger alloy [4]. We found other instances where an existing or new phase nucleates within a precursor coherent precipitate, as shown in Fig. 2 and Fig. 3. Figure 2 shows the new phase called $\zeta$ formed within a spherical coherent precipitate (or Ag GP zone) [5]. This new phase is the three-dimensional version of the Ag-rich interfacial structures observed in some of the strongest Al alloys. Figure 3 shows the nucleation of a disordered $\theta^{\prime}$ phase from the coherent $\theta^{\prime \prime}$ phase under exposure of a scanning probe in the STEM. This suggests that vacancies, such as generated under a high-energy electron beam, are critical to the phase transformations. The high resolution $(\sim 1 \AA)$ and stability in HAADF-STEM mode enabled not only the detailed structures to be determined, but also the quantification of local strain [5]. The results will also be discussed in the light of previous observations on hererophase interfaces [6].

\section{References:}

[1] A Guinier, Nature 142 (1938) p.569; GD Preston, Nature 142 (1938) p.570.

[2] JM Silcock, TJ Heal and HK Hardy, J. Inst. Met. 82 (1953) p.239.

[3] GW Lorimer in "Precipitation Processes in Solids", ed. KC Russell and HI Aaronson, (Met. Soc. AIME) p.115.

[4] Y Chen, Z Zhang, Z Chen, A Tsalanidis, M Weyland, S Findlay, LJ Allen, J Li, NV Medhekar and L Bourgeois, Acta Mater. 125 (2017) p.340. 
[5] Z Zhang, L Bourgeois, JM Rosalie and NV Medhekar, "The Bi-Layered Precipitate Phase $\zeta$ in the Al-Ag System", under review.

[6] L Bourgeois, NV Medhekar, AE Smith, M Weyland, J-F Nie and C Dwyer, Phys. Rev. Lett. 111 (2013) 046102.

[7] The authors acknowledge funding from the Australian Research Council (DP150100558 and LE0454166), computational support from the Monash Sun Grid cluster, the National Computing Infrastructure and Pawsey Supercomputing Centre funded by the Australian Government, and the use of facilities within the Monash Centre for Electron Microscopy.

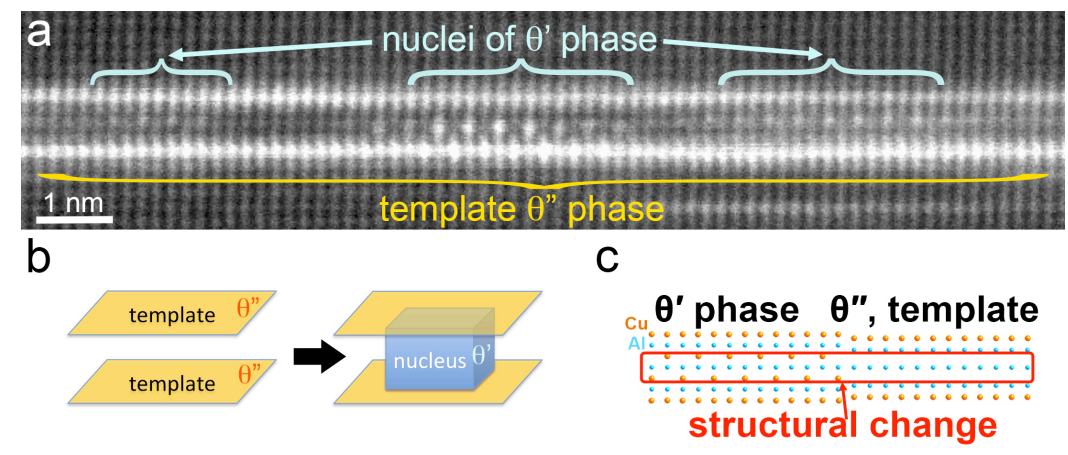

Figure 1. Trace additions of $\mathrm{Au}(200 \mathrm{ppm})$ to the classic alloy $\mathrm{Al}-1.7 \mathrm{at} . \% \mathrm{Cu}$ favour the nucleation of the alloy strengthening phase $\theta^{\prime}$ directly from the coherent precipitate $\theta^{\prime \prime}$ [4], which acts as a template.

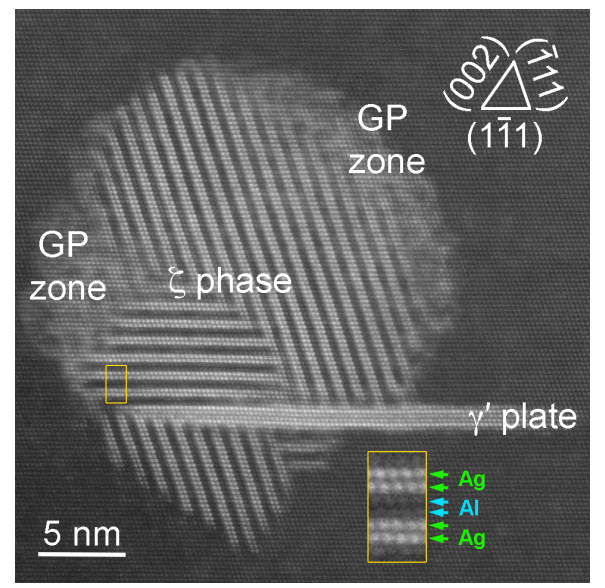

Figure 2. The disordered Ag GP zone in an Al-1.7at.\%Ag alloy can be transformed into the new phase $\zeta$ as well as the known plate-shaped phase $\gamma^{\prime}$.

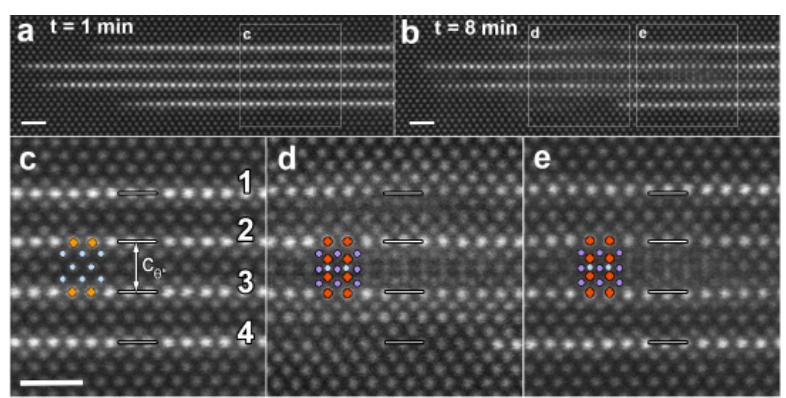

Figure 3. A high-energy $(300 \mathrm{kV})$ STEM probe can bring about the transformation of the coherent phase $\theta^{\prime \prime}$ into a $\theta^{\prime}$-like phase. 